\section{The ring on a finger}

\section{Simon van Heyningen}

THE overwhelming diarrhoea of cholera is produced by a protein toxin secreted by Vibrio cholerae bound to the brush border of the intestine. Some strains of Escherichia coli produce a similar, though less serious disease, and a so-called 'heat-labile toxin' that is essentially the same protein: they are about 80 per cent similar in their amino-acid sequences and have the same mechanism of action. After years of remarkably successful speculation, we now know the structure of the heat-labile toxin from an X-ray diffraction study reported on page 371 of this issue ${ }^{1}$ by Sixma et al. from Groningen. The toxin is made up of two quite different subunits that can fold in and function independently, yet are tightly associated in the intact protein: five copies of the cellbinding $B$ subunit make a ring upon a finger sticking out from the single enzymic A subunit (shown in Fig. 3 of the paper on page 375). No doubt the structure of cholera toxin is the same in all but detail.

Although most of the published work on these proteins was done on cholera toxin, and a preliminary diffraction paper was published 14 years $\mathrm{ago}^{2}$, there was little further progress until the

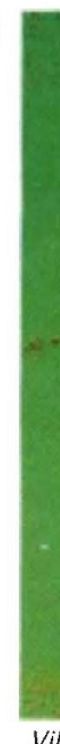
Dutch group decided to concentrate on heatlabile toxin. They made heroic amounts of it, and grew an astonishing array of crystals ${ }^{3}$. Even so, some early crystals were irritatingly non-isomorphous; the group's first useful data sets were collected from the same crystal before and after soaking with heavy metal.

\section{Subunits}

The A and B subunits of these toxins (like similar components of many others) have different tasks. The five B subunits, each of relative molecular mass about $11,600\left(M_{\mathrm{r}}\right.$ $11.6 \mathrm{~K}$ ), bind to ganglioside GM1, which is found in the outer membrane of essentially all eukaryotic cells (the toxins have very little cell specificity). Binding leads to the proteolytic 'nicking' of subunit A into two polypeptide chains, A1 $\left(M_{\mathrm{r}} 22 \mathrm{~K}\right)$ and $\mathrm{A} 2\left(M_{\mathrm{r}}\right.$ $5 \mathrm{~K}$ ), and entry of the A1 chain into the cell. The A.1 polypeptide catalyses the ADPribosylation of the $G$ protein $G_{s \alpha}$, leading to activation of adenylate cyclase, and, presumably, ultimately to the diarrhoea, although the connection has never been established experimentally.

The subunit structure of cholera toxin was determined nearly 20 years ago, and it was clear then from symmetry and other considerations that its five $B$ subunits had to be arranged in a ring with the $\mathrm{A}$ subunit sitting on top of them. Many authors produced diagrams showing such a structure ${ }^{4}$, and more recent support came from twodimensional diffraction experiments $s^{5}$. It is gratifying for traditional protein chemists to see that the old arguments were right, but no one could have predicted the apparently unique interlocking secondary structure.

The ring of B subunits is held together in a way that the authors rightly call "quite spectacular": an individual subunit has two sets

to the membrane, or with the pentamer closest to the membrane and subunit $\mathrm{A}$ on the other side? Most of the evidence ${ }^{5}$ points to the first possibility, in which case the surface of the pentamer that would be on the membrane side looks very suitable because it is flat and highly charged. But Sixma et al. show that the ganglioside-binding sites are not on this flat, charged surface near subunit $A$, but on the other side. Perhaps the toxin does actually bind with subunit A away from the membrane, but that would make it difficult for A to get to the cell surface. Alternatively, if the toxin binds with subunit A downwards, a ganglioside molecule might just be long enough to loop all the way round the outside of the ring while remaining anchored in the membrane. Neither possibility is immediately attractive.

\title{
Enzyme
}

The structure of the A1 peptide would have been worth solving in its own right. It is an enzyme that catalyses a post-translation modification used by many other toxins such as diphtheria and pertussis, as well as by ADP-ribosyltransferases whose function is unknown but which are found in most cells ${ }^{6}$. There are some marked similarities in the active sites of heatlabile toxin and of exoenzyme A of Pseudomonas aeruginos $a^{7}$, the only other such protein whose

of $\beta$ sheet each with three anti-parallel strands, but the first set of one subunit forms a six-stranded sheet with the second set of its neighbour. Each subunit also has a 19residue $\alpha$ helix, arranged so that the five of them line a pore in the middle of the ring, 30 $\AA$ deep and with diameter tapering from $15 \AA$ to $11 \AA$.

Into this pore fits a hairpin loop comprising the $\mathrm{C}$-terminal half of the $\mathrm{A} 2$ peptide (which is known from cross-linking experiments to be involved with binding to the $B$ oligomer). The loop is linked to A1 by the $\mathrm{N}$-terminal half of $\mathrm{A} 2$, a 23-residue helix which holds the globular $\mathrm{A} 1$ peptide and the $B$ pentamer together but slightly apart like an umbilical cord (see Fig. 3 of the paper). The region of $\mathrm{A} 2$ held in the pore is strongly bound to the B pentamer by at least 15 salt bridges. Because five identical subunits bind to a single different subunit, it is hardly surprising that this arrangement is not quite symmetrical. There are many examples of proteins in which association with an asymmetrical partner induces asymmetry in a previously symmetrical molecule.

The mechanism by which $\mathrm{A} 1$ is taken into a cell is not clear; it need not be very efficient because cells can bind millions of toxin molecules, but only a few of A1 are enough to ADP-ribosylate all the $G$ protein. An important question is which way up the toxin binds to a cell - is it with subunit A held close structure has been determined;

\section{toxin.} perhaps they are examples of a new type of $\mathrm{NAD}^{+}$-binding domain characteristic of the ADP-ribosyltransferases. Neither toxin has the well-known 'Rossmann' $\beta \alpha \beta \alpha \beta$ fold characteristic of the $\mathrm{NAD}^{+}$-binding site of dehydrogenases, but this is not surprising because the reaction catalysed by the toxins is quite different.

What good will all this do in South America, Bangladesh and wherever else there is cholera? Sadly, not very much. Protein chemistry might help with the design of better vaccines, but those most at risk from cholera are the poor, to whom even the simple, effective and cheap rehydration therapy ${ }^{8}$ is not always available. What they need is not vaccines, but clean water supplies and better sanitation.

Simon van Heyningen is in the Department of Biochemistry, University of Edinburgh George Square, Edinburgh EH8 9XD, UK.

\footnotetext{
1. Sixma, T. K. et al. Nature 351, 371-377 (1991).

2. Sigler, P. S., Druyan, M. E., Kiefer, M. C. \& Finkelstein, R. A. Science 197, 1277-1279 (1977).

3. Pronk, S. E. etal. J. biol. Chem. 260, 13580-13584(1985).

4. van Heyningen, S. Biol. Rev. 52, 509-549 (1977).

5. Ribi, H. O., Ludwig, D. S., Mercer, K. L., Schoolnik, G. K. \& Kornberg, R. D. Science 239, 1272-1324 (1988).

6. Moss, J. \& Vaughan, M. (eds) ADP-ribosylating Toxins and $G$ Proteins: Insights into Signal Transduction (Am. Soc. Micro biol., Washington, 1990).

Brandhuber, B. J., Allured, V. S., Faibel, T. G. \& Mckay, D. B. Proteins 3, 146-154 (1988).

Proteins 3 , 14 8. Hirschhorn, N. \& Greenough. W. B. Sci. Am. 264, 16-22 (1991)
} 